\title{
Músculo Aductor Mínimo en Individuos Brasileños
}

\author{
Adductor Minimus Muscle in Brazilian Individuals
}

\author{
Luiz Carlos Buarque De Gusmão*; Jacqueline Silva Brito Lima**; Marcos Sergio De Lima Santos***; \\ Ralmony De Alcantara Santos ${ }^{* * * *} \&$ Camila Carvalho Cavalcante ${ }^{* * * * *}$
}

GUSMÃO, L. C. B.; BRITO LIMA, J. S.; LIMA SANTOS, M. S.; SANTOS, R. A. \& CAVALCANTE, C. C. Músculo aductor mínimo en individuos brasileños. Int. J. Morphol., 32(2):440-443, 2014.

RESUMEN: Entre el grupo muscular que realiza la aducción de la articulación del cuadril se destaca el músculo aductor magno, cuya porción superior de la parte aductora puede constituir el músculo aductor mínimo. En la literatura hay pocas citaciones sobre este músculo, más allá de la presencia de datos divergentes entre diversos autores. De este modo, el objetivo de este estudio ha sido el de analizar el músculo aductor mínimo con relación: a sus inserciones proximal y distal, a las disposiciones de las fibras musculares y establecer la incidencia del mismo. Cincuenta miembros inferiores aislados de cadáveres adultos de ambos sexos fueron disecados. Los resultados encontrados demuestran que el músculo fue observado en dieciséis casos (32\%), presentando: inserción proximal predominante en el ramo isquiopubiano (37,5\%), e inserción distal en la línea áspera del fémur (100\%). Las fibras musculares estaban dispuestas de forma oblicua en catorce casos $(87,5 \%)$ y con disposición transversal en apenas dos casos (12,5\%). Por lo tanto, este estudio servirá como base para nuevos trabajos científicos sobre el músculo aductor mínimo, con el objeto de clasificarlo como un músculo aislado.

PALABRAS CLAVE: Miembro inferior; Muslo; Anatomía.

\section{INTRODUCCIÓN}

Los músculos del compartimiento medial del muslo son colectivamente denominados de grupo aductor, puesto que realizan primordialmente la aducción del cuadril y, consecuentemente, del miembro inferior (Moore \& Dalley, 2007; Dangelo \& Fattini, 2007). En esta musculatura se destaca el músculo aductor magno, considerado el más potente y el de mayor masa muscular (Woodburne, 1984; Gardner et al., 1988; Palastanga et al., 1998). El músculo aductor magno posee funciones de aducción y extensión, siendo la parte aductora inervada por el nervio obturador y la parte extensora por la parte tibial del nervio isquiático (Goss, 1988; Palastanga et al.; Putz \& Pabst, 2006). Más allá de los movimientos de aducción y extensión, este músculo también puede realizar rotación lateral cuando el cuadril se encuentra aducido (Floyd \& Thompson, 2002; Hall, 2005).

Le Double (1897), Woodburne e Goss describen que las fibras musculares más superiores, aductoras, son horizontales; y las inferiores, extensoras, son verticales. Testut (1884), Schaeffer (1942), Benninghoff \& Goerttler (1980) y Gardner et al. explican que las fibras musculares horizon- tales superiores de la parte aductora pueden presentar una separación, originando un pequeño músculo.

Sin embargo, a pesar de que su existencia es ignorada por algunos autores (Rauber \& Kopsch, 1947; Clemente, 1981), Le Double relata que de 6 en 10 individuos la porción superior del aductor magno está separada enteramente de la porción inferior, de manera que esa porción superior o transversa ha sido llamada de cuarto aductor por Diemerbrock y de aductor mínimo por Günther. En un estudio realizado con 40 miembros inferiores de cadáveres adultos, el músculo aductor mínimo fue encontrado en $52,5 \%$ de los casos (Shane Tubbs et al., 2011). El músculo aductor mínimo, cuando presente, posee el mismo pedículo vascular que se distribuye al músculo grácil (DiGeronimo, 1982), siendo inervado por el nervio obturador y con inserción proximal en el pubis (Prives et al., 1971). No obstante, Schaeffer, Hamilton (1976), Gardner et al., Goss y Spalteholz \& Spanner (1988) relatan que esa inserción seria en el ramo isquiopubiano. Con respecto a la inserción distal, Testut, Schaeffer, Prives et al. y Gardner et al., explican que esta se

\footnotetext{
* Máster y Doctor en Anatomía Humana; Profesor de la Universidad Federal de Alagoas; Miembro Titular del Colegio Brasileño de Cirujanos.

** Máster en Anatomía Humana, Profesora de la Universidad Federal de Alagoas, Maceió, Brasil.

*** Profesores de Educación Física, Maceió, Brasil.

***** Monitora de la Asignatura Anatomía Humana de la Universidad Federal de Alagoas, Maceió, Brasil.
} 
realiza en la línea áspera del fémur. Woodburne y Goss incluyen, además de la línea áspera, la tuberosidad del trocánter menor del fémur.

El músculo aductor mínimo fue utilizado en la reconstrucción escrotal, sirviendo como una alternativa de colgajo miocutáneo en pacientes que presentan extensa lesión tisular en región perineal (DiGeronimo). Debido a la presencia de datos divergentes encontrados en la literatura pesquisada, los autores buscaron aclarar y fornecer datos anatómicos en relación al músculo aductor mínimo en individuos brasileños, evaluándolo con respecto a su incidencia, inserciones y disposición de sus fibras musculares.

\section{MATERIAL Y MÉTODO}

Cumpliendo lo que determina la Ley Federal $n^{\circ} 8.501$, del 30 de noviembre de 1992, y tras aprobación institucional, fueron utilizados 50 miembros inferiores aislados de cadáveres adultos de ambos sexos, fijados en formaldehido a $10 \%$, pertenecientes al laboratorio de Anatomía de la Universidad Federal de Alagoas.

En cada miembro fue disecado el músculo aductor magno y verificada la existencia del músculo aductor mínimo. En caso de presencia, este fue evaluado en relación a su inserción proximal y distal, como así también a la disposición de sus fibras musculares.

\section{RESULTADOS}

En los 50 miembros inferiores disecados fue posible observar el músculo aductor mínimo en 16 casos (32\%) (Fig. 1). En relación a la zona de inserción proximal de este músculo, ha sido constatado que hubo predominancia en el ramo isquiopubiano, que estuvo presente en 6 casos $(37,5 \%)$ (Fig. 2), seguido por la porción inferior de la tuberosidad isquiática presente en 5 casos $(31,25 \%)$. En 4 casos (25\%) se verificó la inserción en el ramo inferior del pubis, y en apenas 1 caso $(6,25 \%)$ aquella ocurrió en el ramo del isquion. Al analizar su inserción distal, fue averiguado que todos los casos (100\%) presentaron inserción en el tercio proximal de la línea áspera del fémur (Fig. 3).
Con respecto a la disposición de las fibras musculares del músculo aductor mínimo, se detectó que 14 casos (87,5\%) presentaron disposición oblicua (Fig. 3) y en dos casos (12,5\%) las fibras estaban dispuestas transversalmente.

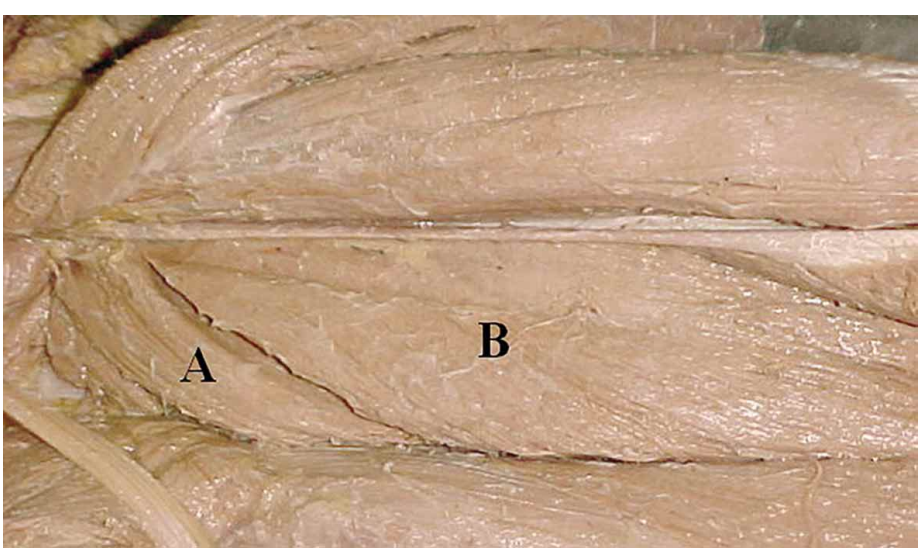

Fig. 1. Fotografía del muslo izquierdo; A. Músculo aductor mínimo; B. Parte aductora del músculo aductor magno.

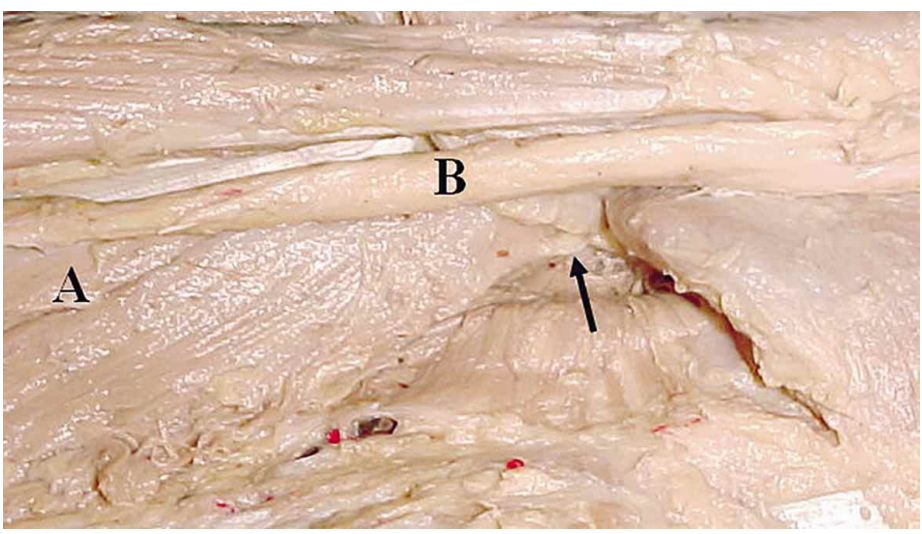

Fig. 2. Fotografía del muslo derecho; La flecha representa el origen del músculo aductor mínimo; A. Parte aductora del músculo aductor magno. B. Nervio isquiático.

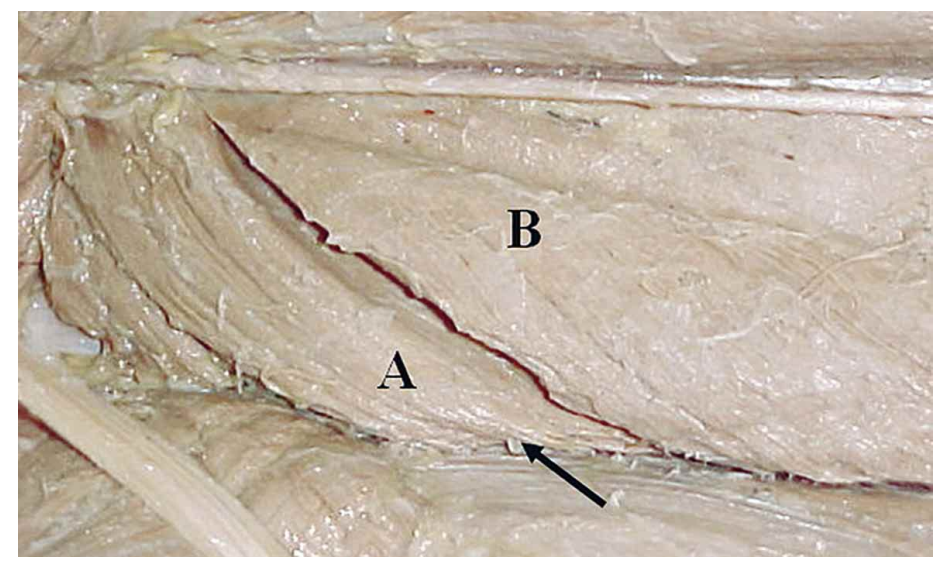

Fig. 3. Fotografía del muslo izquierdo; La flecha representa la inserción distal en la línea áspera del fémur; A. Disposición oblicua de las fibras musculares del músculo aductor mínimo; B. Parte aductora del músculo aductor magno. 


\section{DISCUSIÓN}

El músculo aductor mínimo estuvo presente en 16 casos, correspondiendo a $32 \%$ de la muestra analizada, siendo este resultado inferior al obtenido por Le Double (60\%) y Shane Tubbs $(52,5 \%)$.

De acuerdo con la disposición de las fibras encontradas, ha sido constatado que en $87,5 \%$ de los casos, el músculo analizado poseía fibras musculares oblicuas en toda su extensión, caso este que contradice a Testut, Schaeffer, Benninghoff \& Goerttler y a Gardner et al., quienes relatan que estas fibras musculares serían horizontales en vez de oblicuas. No obstante, es importante resaltar que esa disposición horizontal también fue encontrada en nuestro estudio, representando $12,5 \%$ de los casos.

En nuestros hallazgos, la inserción proximal del músculo aductor mínimo se realiza predominantemente en el ramo isquiopubiano, correspondiendo a $37,5 \%$ de los ca- sos, concordando con Schaeffer, Hamilton, Gardner et al., Goss y Spalteholz \& Spanner.

Con relación a la inserción distal, ha sido verificado que en $100 \%$ de los casos el músculo mencionado presentó inserción en el tercio proximal de la línea áspera del fémur, confirmando los hallazgos encontrados por Testut, Schaeffer, Prives et al. y Gardner et al., sin embargo, al contrario de Woodbum y Goss, no ha sido observada ninguna inserción distal en la tuberosidad del trocánter menor del fémur en nuestra muestra.

Tras el análisis de los resultados obtenidos, se puede concluir que la incidencia del músculo aductor mínimo fue relevante, lo que permite clasificarlo como un cuarto aductor. Por otro lado, las informaciones anatómicas presentadas refuerzan la indicación del músculo en las cirugías reconstructoras del perineo.

GUSMÃO, L. C. B.; BRITO LIMA, J. S.; LIMA SANTOS, M. S.; SANTOS, R. A. \& CAVALCANTE, C. C. Adductor minimus muscle in Brazilian individuals. Int. J. Morphol., 32(2):440-443, 2014.

SUMMARY: Among the muscle group that performs the adduction of the hip joint, there is the adductor magnus muscle which upper portion of the adductor part may constitute the adductor minimus muscle. In the literature there are few quotes on this muscle, besides the presence of conflicting data among several authors. Thus, the objective of this study was to analyze the adductor minimus muscle in respect to: its proximal and distal insertions, the provisions of its muscle fibers and establish the incidence of it. Fifty lower limbs isolated from adult cadavers of both sexes were dissected. The results showed that the muscle was visualized in sixteen cases (32\%), with proximal insertion predominant in ischiopubic ramus (37.5\%), and distal insertion in the linea aspera of the femur (100\%). The muscle fibers were arranged obliquely in fourteen cases (87.5\%) and in only 2 cases $(12.5 \%)$ the fibers were arranged transversely. Therefore, this study will serve as a foundation for new scientific work on the adductor minimus muscle, in order to classify it as an isolated muscle.

KEY WORDS: Lower limb; Thigh; Anatomy.

\section{REFERENCIAS BIBLIOGRÁFICAS}

Benninghoff, H. F. \& Goerttler, J. S. Trattato di anatomia umana funzionale. $2^{\mathrm{a}}$ ed. Padova, Piccin, 1980.

Clemente, C. D. Anatomy: A regional atlas of the human body. $2^{\text {nd }}$ ed. Baltimore, Urban \& Schwarzenberg, 1981.

Dangelo, J. G. \& Fattini, C. A. Anatomia humana sistêmica e segmentar. $3^{\text {a }}$ ed. São Paulo, Atheneu, 2007.

DiGeronimo, E. M. Scrotal reconstruction utilizing a unilateral adductor minimus myocutaneous flap. Plast. Reconst. Surg., 70(6):749-51, 1982.

Floyd, R. T. \& Thompson, C. W. Manual de cinesiologia estrutural. $14^{\mathrm{a}}$ ed. São Paulo, Manole, 2002.
Gardner, E.; Gray, D. J. \& O’ Rahilly, R. Anatomia: estudo regional do corpo humano. $4^{\mathrm{a}}$ ed. Rio de Janeiro, Guanabara Koogan, 1988.

Goss, C. M. Gray Anatomia. 29a ed. Rio de Janeiro, Guanabara Koogan, 1988.

Hall, S. J. Biomecânica Básica. $4^{\mathrm{a}}$ ed. Rio de Janeiro, Guanabara Koogan, 2005.

Hamilton, W. J. Textbook of human anatomy. $2^{\text {nd }}$ ed. London, Macmillan, 1976.

Le Double, A. F. Traite des variations du systeme musculaire de l'homme et de leur signification au point de vue de 
GUSMÃO, L. C. B.; BRITO LIMA, J. S.; LIMA SANTOS, M. S.; SANTOS, R. A. \& CAVALCANTE, C. C. Músculo aductor mínimo en individuos brasileños. Int. J. Morphol., 32(2):440-443, 2014.

l'anthropologie zoologique. Paris, Schleicher Freres, 1897.

Moore, K. L. \& Dalley, A. F. Anatomia orientada para clínica. $5^{\mathrm{a}}$ ed. Río de Janeiro, Guanabara Koogan, 2007.

Palastanga, N.; Field, D. \& Soames, R. Anatomy \& Human Movement: Structure \& Function. $3^{\text {rd }}$ ed. Boston, Butterworth Heinemann, 1998.

Prives, M.; Lisenkov, N. \& Bushkovich, V. Anatomia humana. $3^{\mathrm{a}}$ ed. Moscú, Mir, 1971.

Putz, R. \& Pabst, R. Sobotta: atlas de anatomia humana. $22^{\mathrm{a}} \mathrm{ed}$. Río de Janeiro, Guanabara Koogan, 2006.

Rauber, A. \& Kopsch, F. Lehrbuch und Atlas der Anatomie des Menschen. $17^{\text {th }}$ ed. Leipzig, Thieme, 1947.

Schaeffer, J. P. Morris' human anatomy: a complete systematic treatise. $11^{\text {th }}$ ed. New York, Eleventh, 1942.

Spalteholz, W. \& Spanner, R. Atlas de anatomia humana. São Paulo, Roca, 1988.

Shane Tubbs, R.; Griessenauer, C. J.; Marshall, T.; Dennison, C. P.; Shoja, M. M.; Loukas, M.; Apaydin, N. \& Cohen-Gadol, A. A. The adductor minimus muscle revisited. Surg. Radiol. Anat., 33(5):429-32, 2011.

Testut, L. Les Anomalies Musculaires Chez l'Homme Expliquèes par l'Anatomie Compare et Leur Importance en Anthropologie. Paris, Masson, 1884.

Woodburne, R. T. Anatomia Humana. $6^{\mathrm{a}}$ ed. Río de Janeiro, Guanabara Koogan, 1984.

\author{
Dirección para Correspondencia: \\ Prof. Dr. Luiz Carlos Buarque de Gusmão \\ Condominio Aldebaran Alfa \\ Av. Helio Castro Vasconcelos - $s / n$ \\ Quadra F, nº 08. \\ Bairro: Jardim Petrópolis \\ CEP: $57080-545$ \\ Maceió-AL \\ BRASIL
}

Email: camila_ccavalcante@hotmail.com

Recibido : 22-09-2013

Aceptado: 18-02-2014 